DOI: 10.12731/2227-930X-2019-4-7-10 УДК 656.1

\title{
ЗАДАЧИ КОММИВОЯЖЕРА ПРИ ОПТИМИЗАЦИИ МАРШРУТНОГО ПУТИ
}

\author{
Azаnoва Е.Г., Попова T.M.
}

В статье изучены вопросы оптимизации мармрутного пути, специфики дорожного движения в городе с учетом потери времени на заторы на дорогах. Предложен оптимальный марирут торгового представителя, который задает последовательность всех торговых точек и описывает оптимальные пути следования от одной торговой точки к другой.

Ключевые слова: управление перевозками; задачи коммивояжера; алгоритм Дейкстры; временной параметр; оптимизация марирута.

\section{CHALLENGES OF THE TRAVELER IN THE OPTIMIZATION OF THE ROUTE WAY}

\section{Agapova E.G., Popova T.M.}

The article studies the optimization of the route, the specifics of traffic in the city, taking into account the loss of time for traffic jams. The optimal route of the sales representative is proposed, which sets the sequence of all outlets and describes the optimal route from one outlet to another.

Keywords: traffic management; traveling salesman tasks; Dijkstra's algorithm; time parameter; route optimization.

Специфика работы торгового представителя состоит в постоянном перемещении от одной точки к другой, что занимает определенное количество времени. Количество точек посещения может достигать тридцати в день, а то более. Это зависит от загруженности рабочего дня, количества запланированных посещений. В современных условиях дорожного движения при перемещении от одной точки к другой возникают определенные трудности: как расплани- 
ровать рабочий день так, чтобы посетить все торговые точки; какой путь выбрать при перемещении от одной точки до другой, чтобы это занимало наименьшее количество времени; выбрать начальную точку отправления для удобства перемещения и прийти к конечной точке пути. Данный вопрос рассматривается с точки зрения подхода к математическому моделированию в управлении процессами перевозок, приводится классификация моделей, применяемых при управлении процессами перевозок и требования к ним [1, с. 43-45].

Для конкретной рассматриваемой задачи рассматривается множество торговых точек (вершин), которые распределены на неделю (в каждый конкретный день недели определенные торговые точки в определенном количестве). Заданный граф накладывается на карту города Хабаровска. Также заданы все пути перемещения между торговыми точками с учетом специфики дорожного движения на данной территории. Город поделен на территории, закрепленные за конкретным работником предприятия. Каждая закрепленная территория имеет определенное количество торговых точек, которые распределены по дням недели. Предполагается, что рабочая неделя состоит из пяти рабочих дней, причем торговые точки понедельника соответствуют торговым точкам четверга, торговые точки вторника - пятнице. Торговые точки среды не повторяются.

Итак, начальная и конечная вершины графа совпадают, то есть коммивояжер (торговый представитель) должен выехать из исходного пункта «0» и вернутся в него же, объехав весь маршрут за минимальное время.

Представим в виде таблицы расстояния (длины дуг) между пунктами назначения. Номер строки - начало дуги, номер столбца - конец дуги. На пересечении - расстояние между двумя точками или вес дуги. Итак, нам дан ориентированный граф (адреса торговых точек). Данный граф невозможно изобразить графически, так как он содержит большое количество дуг. Каждая вершина графа (кроме вершины 0 начальной и конечной вершины) является и началом, и концом дуги, из чего вытекает огромное количество вариантов обхода данного графа.

Алгоритм ближайшего соседа является самым простым вариантом решения задачи, если не заданы дополнительные параметры. 
Трудность данного алгоритма состоит в том, что требуется перебрать большое количество вариантов, а их может быть великое множество, что не позволит выделить единственное оптимальное решение. Итак, коммивояжер отправляется из вершины 0. По таблице довольно просто определить его ближайшего «соседа». В конце маршрута требуется вернуться в вершину 0 , обойдя все вершины. Здесь учитывается только километраж.

В случае задачи о коммивояжере эта идея представления задачи в виде многошагового процесса реализуется следующим образом. Отметим среди n точек, которые нужно обойти, произвольную точку, которую будем считать точкой старта и финиша (вершина 0). Первым этапом принятия решения будем считать выбор первой точки, в которую надо идти из места старта, вторым этапом - выбор следующей точки и так далее.

По алгоритму задачи коммивояжера с временным параметром в качестве параметра рассмотрим время, которое может зависеть о многих факторов: пробки на дорогах, качество дорожного покрытия, дорожные работы, светофоры, плотность потока автомобилей (а в данной задаче предполагается передвижение от точки до точки именно на автомобиле), время года и так далее.

Разработанная математическая модель имеет тенденцию развития в будущем, так как дорожная система города развивается, появляются новые дороги и новые пути к достижению цели.

\section{Список литературы}

1. Абульманова К.И., Наконечный В.Н., Савкина Т.Б., Туркот Ю.В. К вопросу применения математических моделей в управлении процессами перевозок // В сборнике: Труды международной научно-практической конференции «Транспорт-2015» ФГБОУ ВПО «Ростовский государственный университет путей сообщения». 2015. С. 43-45.

\section{References}

1. Abul'manova K.I., Nakonechnyj V.N., Savkina T.B., Turkot Yu.V. K voprosu primeneniya matematicheskih modelej $\mathrm{v}$ upravlenii processami perevozok //V sbornike: Trudy mezhdunarodnoj nauchno-prakticheskoj 
konferencii «Transport-2015» FGBOU VPO «Rostovskij gosudarstvennyj universitet putej soobshcheniya». 2015. S. 43-45.

\section{ДАННЫЕ ОБ АВТОРАХ}

Агапова Елена Григорьевна, доцент, кандидат физико-математических наук

Тихоокеанский государственный университет

ул. Тихоокеанская, 136, г. Хабаровск, 680035, Российская Федерачия

000614@pnu.edu.ru

Попова Татьяна Михайловна, доцент, кандидат физико-математических наук

Тихоокеанский государственный университет

ул. Тихоокеанская, 136, г. Хабаровск, 680035, Российская Федерачия

000511@pnu.edu.ru

\section{DATA ABOUT THE AUTHORS}

Agapova Elena Grigoryevna, associate professor, candidate of physical and mathematical sciences

Pacific National University

136, Tikhookeanskaya Str, city of Khabarovsk, 680035, Russian Federation

000614@pnu.edu.ru

ORCID: 0000-0002-2824-6294

Popova Tatyana Mikhajlovna, associate professor, candidate of physical and mathematical sciences

Pacific National University

136, Tikhookeanskaya Str, city of Khabarovsk, 680035, Russian Federation

000511@pnu.edu.ru

ORCID: 0000-0003-4759-9500 